\title{
Laparoscopic Left Hepatectomy for Ruptured Hepatocellular Carcinoma Controlled After Transcatheter Arterial Embolization: Case Report and Review of the Literature
}

\author{
SHOHEI YOSHIYA ${ }^{1,2}$, KENTARO IWAKI $^{1}$, AKIHIRO SAKAI $^{1,2}$, SHUNSUKE FUJITA ${ }^{1}$, \\ TAKAHIDE KAWASAKI ${ }^{1}$, FUMITAKA YOSHIZUMI ${ }^{1}$, SHOJI HIROSHIGE ${ }^{1,2}$, MASAHIRO OKAMOTO $^{1,2}$, \\ KENGO FUKUZAWA $^{1,2}$, AKIRA MOTOHIRO ${ }^{1,2}$ and YOSHIHIKO MAEHARA ${ }^{2}$ \\ ${ }^{1}$ Department of Surgery, Oita Red Cross Hospital, Oita, Japan; \\ ${ }^{2}$ Department of Surgery and Science, Graduate School of Medical Sciences, Kyushu University, Fukuoka, Japan
}

\begin{abstract}
Background: Although spontaneous tumour rupture is a life-threatening complication of hepatocellular carcinoma (HCC), staged hepatectomy for HCC controlled after transcatheter arterial embolization (TAE) could provide a better prognosis. Laparoscopic liver resection (LLR) has been accepted worldwide and has been expanded from minor resection to anatomical major resection. We herein report the first case of pure laparoscopic left hepatectomy for ruptured HCC controlled after TAE. Case Report: A 66-year-old man was transferred to our Institute because of abdominal pain and decreased consciousness. Ruptured HCC in segment IV and massive intra-abdominal haemorrhage were diagnosed. Emergency TAE was performed, achieving haemostasis. Reduction of intra-abdominal haemorrhage was confirmed at the 3-month follow-up, and no intrahepatic metastasis or peritoneal dissemination was present. Therefore, we performed elective laparoscopic left hepatectomy for the remaining HCC 110 days after TAE. Although dense adhesion was found in the upper right peritoneal cavity and greater omentum enveloping the remaining haemorrhage on the underside of the liver, there was no disseminated involvement in the peritoneal cavity. The operative time was 194 minutes, and intraoperative blood loss was $100 \mathrm{~g}$. The postoperative course was uneventful, and the patient was discharged on postoperative day 6. Conclusion: Major LLR
\end{abstract}

This article is freely accessible online.

Correspondence to: Shohei Yoshiya, MD, Ph.D., Department of Surgery, Oita Red Cross Hospital, 3-2-37, Chiyo-machi, Oita, Oita 870-0033, Japan. Tel: +81 975326181, Fax: +81 975331207, e-mail: yoshiya@surg2.med.kyushu-u.ac.jp

Key Words: Laparoscopic hepatectomy, laparoscopic liver resection, left lobectomy, ruptured HCC, TAE. may be an option for staged hepatectomy in patients with ruptured HCC controlled after TAE.

Spontaneous tumour rupture is a life-threatening complication of hepatocellular carcinoma (HCC). The reported incidence of $\mathrm{HCC}$ rupture is $3 \%$ to $26 \%$, and $\mathrm{HCC}$ rupture is associated with a high rate of in-hospital death (1). Some studies have shown that staged hepatectomy for ruptured HCC leads to favourable surgical outcomes $(1,2)$. However, laparoscopic liver resection (LLR) has been accepted worldwide and expanded from minor resection to anatomical major resection still in an exploratory or learning phase (3). We herein report a case of pure laparoscopic left hepatectomy for ruptured HCC controlled after transcatheter arterial embolization (TAE). To our knowledge, this is the first case report of laparoscopic hepatic lobectomy for spontaneous ruptured HCC controlled after TAE.

\section{Case Report}

A 66-year-old man was transferred to our Institute because of abdominal pain and decreased consciousness. His vital signs were stable, but he had muscular guarding. Blood examination showed a low haemoglobin level $(10.6 \mathrm{~g} / \mathrm{dl})$ and negativity for hepatitis B virus surface antigen and hepatitis $\mathrm{C}$ virus antibody. Enhanced computed tomography revealed a 6-cm mass with a high-low pattern in segment IV (S4) of the liver and fluid collection in the peritoneal cavity, especially around the liver. We diagnosed a ruptured HCC in $\mathrm{S} 4$ with massive intra-abdominal haemorrhage (Figure 1A). Emergency angiographic examination showed that the middle hepatic artery and S4 branch of the left hepatic artery were the feeding arteries of the ruptured HCC. TAE with a gelatin sponge for both feeding arteries was performed and arrested haemorrhage. The patient developed no complications after TAE and was discharged on hospital day 6. 

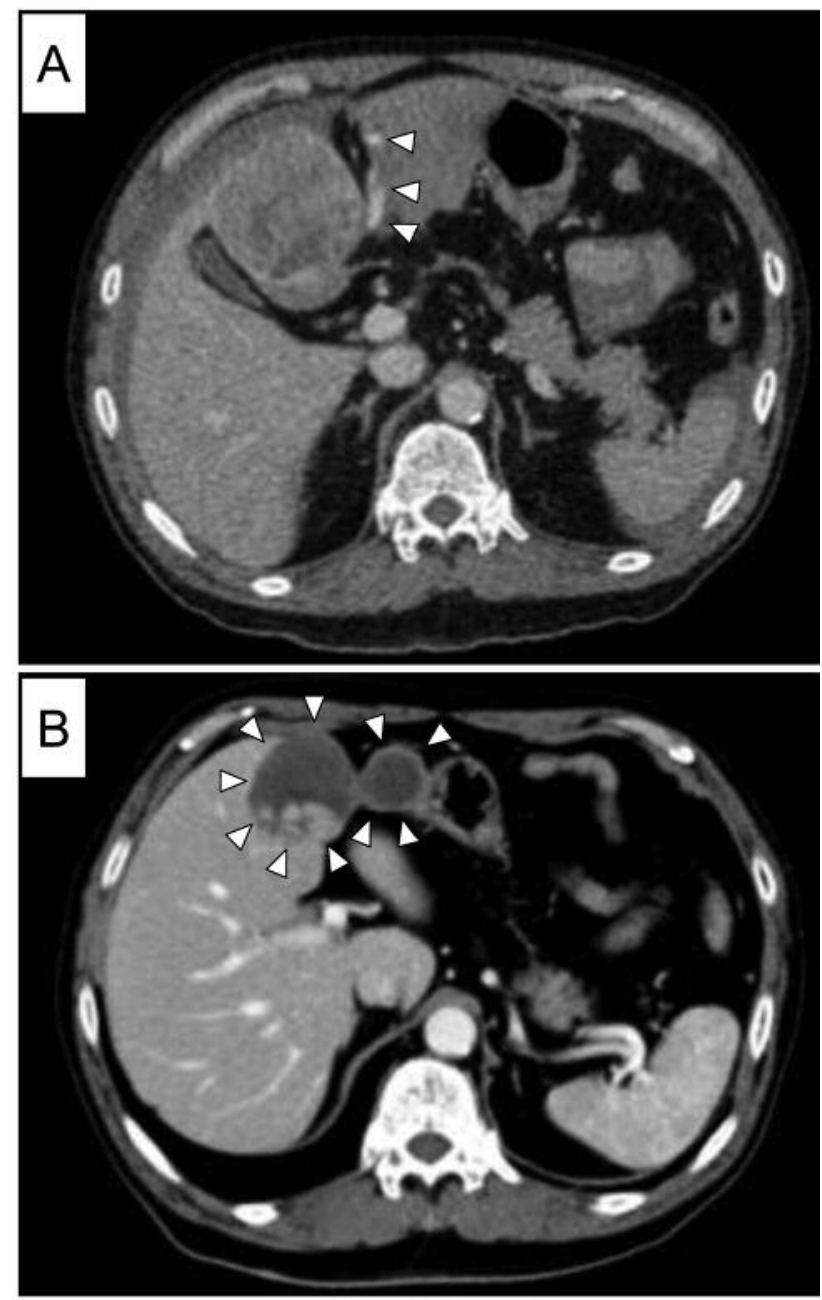

Figure 1. A: Portal phase of enhanced computed tomography at the time of spontaneous tumour rupture. A 6-cm hepatocellular carcinoma was present in segment 4 of the liver, and fluid collection was present in the peritoneal cavity. The arrowheads indicate extravasation of haemorrhage. B: Enhanced computed tomography 3 months after transcatheter arterial embolization revealed a reduction in the volume of the main tumour and intra-abdominal haemorrhage (arrowheads) and no intrahepatic metastasis or peritoneal dissemination. Adhesion between the remaining haemorrhage and stomach was suspected.

Examination at the 3-month follow-up confirmed the reduction of intra-abdominal haemorrhage without intrahepatic metastasis or peritoneal dissemination (Figure 1B). Liver functional tests revealed the following: albumin, $4.0 \mathrm{~g} / \mathrm{dl}$; total bilirubin, $0.49 \mathrm{mg} / \mathrm{dl}$; platelets, $17.0 \times 104 / \mu \mathrm{l}$; prothrombin time-international normalized ratio, 0.99; and indocyanine green retention rate at $15 \mathrm{~s}, 8.8 \%$. Therefore, we performed elective laparoscopic left hepatectomy for the remaining HCC.

The laparoscopic surgery was performed 110 days after TAE. A 12-mm trocar was inserted at the umbilicus with an open
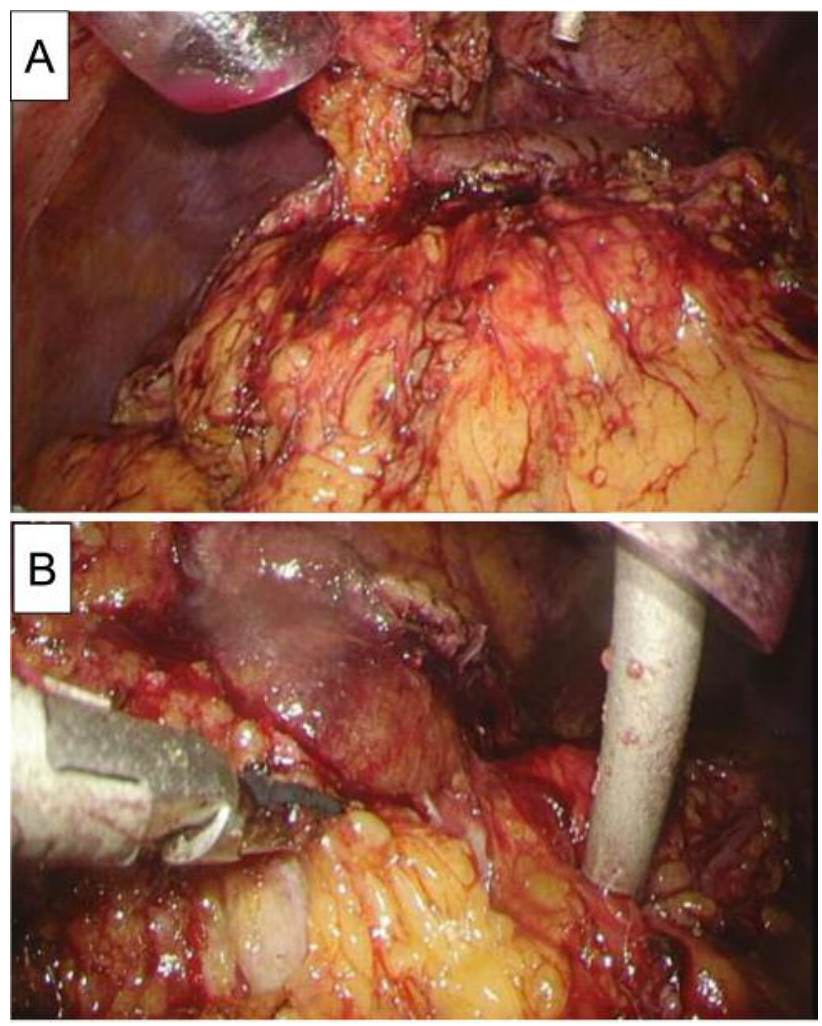

Figure 2. Intraoperative findings. A: Dense adhesion was detected in the upper right peritoneal cavity, and the greater omentum enveloped the remaining haemorrhage and main tumour at the hepatic underside. $B$ : Close adhesion was present among the main tumour, remaining haemorrhage, and stomach.

method involving insertion of a camera port and establishment of pneumoperitoneum. Dense adhesion was detected in the upper right peritoneal cavity, and the greater omentum enveloped the remaining haemorrhage and main tumour at the hepatic underside (Figure 2). No laparoscopic disseminated involvement was present in the peritoneal cavity, hence we decided to perform radical surgery. Four 12-mm working trocars were also inserted at the epigastrium, both sides of the hypochondrium, and right lateral region. Firstly, we attempted to carefully divide the adhesion in the upper right peritoneal cavity and resect part of the greater omentum, which was solidly adhered to the haemorrhage site, for en bloc extirpation. Adhesiolysis required a long period of time but was successfully accomplished laparoscopically. Next, we mobilized the left liver and performed Pringle's manoeuvre. Hepatic parenchymal transection was performed using an ultrasonic dissector (CUSA ${ }^{\mathrm{TM}}$; Integra LifeSciences Corp., Plainsboro, NJ, USA), the clamp-crashing technique with the HARMONIC ACETM+7 (Ethicon Endo-Surgery, Cincinnati, OH, USA), and a soft-coagulation system with Pringle's manoeuvre (total of 54 


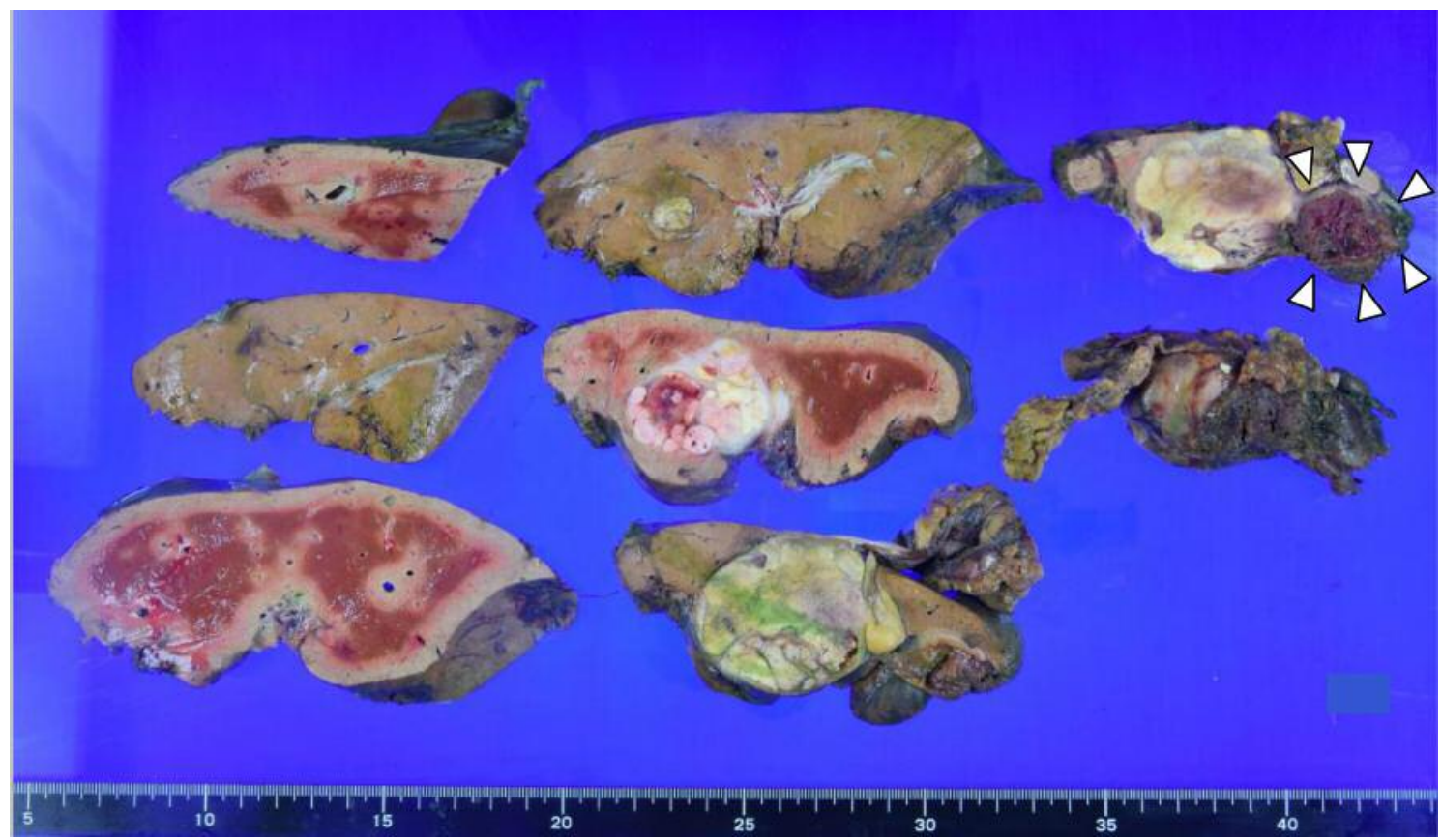

Figure 3. The resected liver volume was $356 \mathrm{~g}$ and the tumour size was $46 \times 47 \mathrm{~mm}$. The arrowheads indicate the haemorrhage remaining close to the main tumour.

minutes). Small vessels were sealed with the HARMONIC, and large vessels, including V4 and branches of the Glissonian pedicle, were sealed with clips. The left Glissonian pedicle and left hepatic vein were divided with Endo GIATM Stapler with Tri-Staple $^{\mathrm{TM}}$ Technology (Medtronic, Minneapolis, MN, USA). The resected specimen was extracted through the umbilical trocar site, which was expanded to $6 \mathrm{~cm}$. The operative time was 194 minutes, intraoperative blood loss was $100 \mathrm{~g}$, and resected liver volume was $356 \mathrm{~g}$ (Figure 3).

The postoperative course was uneventful, and the patient was discharged on postoperative day 6. Pathological examination revealed moderately differentiated HCC, trabecular type, grade 2, tumour size $46 \times 47 \mathrm{~mm}$, with intrahepatic metastasis, no invasion of portal vein, hepatic vein or artery, nor of the bile duct, and negative surgical margins.

\section{Discussion}

Spontaneous tumour rupture is a life-threatening complication that accounts for $6 \%$ to $10 \%$ mortality in patients with $\mathrm{HCC}(4$, $5)$. Recent studies have shown that TAE can effectively induce haemostasis with a success rate of approximately $100 \%(1,6)$. Tanaka et al. reported that emergency hepatic resection was an independent risk factor for in-hospital death (1); therefore, TAE is considered the first treatment option for ruptured HCC. Although patients with ruptured $\mathrm{HCC}$ have worse survival than patients with non-ruptured HCC because of more advanced tumour stages, as reflected by the tumour size, tumour number, vascular invasion, and higher tumour marker levels (7), recent studies have demonstrated that rupture itself is not a significant risk factor for unfavourable surgical outcomes in patients who have undergone complete resection for ruptured HCC or patients who did not die in hospital $(1,2,6)$. Additionally, Aoki et al. found that the prognosis of patients who had undergone staged hepatectomy was significantly better than that of patients who had undergone transcatheter arterial chemoembolization, chemotherapy, or best supportive care (with 1-, 3-, and 5-year overall survival rates in the staged hepatectomy group of $76.0 \%$, $48.6 \%$, and $33.9 \%$, and in the TACE group of $39.7 \%, 14.1 \%$, and $6.0 \%$, respectively) (7). Because peritoneal recurrence is a rare event, complete resection of ruptured HCC may lead to long-term survival (2). Therefore, patients with good liver function for complete resection after ruptured HCC are preferable for staged hepatectomy.

A recent study showed that the duration from TAE to staged hepatectomy was 40 days (range $=0-129$ days) (1); this result was obtained because re-rupture after TAE for initial rupture occurred mainly within 1 month (8). On the other hand, the time to surgery was 110 days. This longer interval led to reduced intraperitoneal haemorrhage. This reduction in haemorrhage volume might enable the accomplishment of pure laparoscopic left hepatectomy. Furthermore, during this interval, no intrahepatic metastasis or peritoneal dissemination was revealed. 
LLR has been expanded to include minor resection, major resection, anatomical resection, donor hepatectomy (3), hepatectomy with thrombectomy (9), and radical resection for hilar cholangiocellular carcinoma (10). Moreover, recent studies have revealed the usability and safety of LLR in patients with liver cirrhosis (11) and those undergoing repeat hepatectomy (12). However, the Second International Consensus Conference on Laparoscopic Liver Surgery recommended that major LLR is an innovative procedure and still in exploration or learning phase (3). Therefore, the indications for major LLR should be strict. In the present case, the tumour was located in S4 of the liver, and the remnant haematoma was close to S4 and the left lateral segment; thus, left hepatectomy was needed as en bloc resection. Fortunately, we have extensive experience with many minor and major LLR procedures; therefore, we elected to perform pure laparoscopic left hepatectomy as staged hepatectomy and achieved a good postoperative course for the patient.

We consider that the benefit of staged hepatectomy for ruptured HCC after TAE is the laparoscopic view itself. Regardless of whether a careful preoperative examination has been performed, peritoneal dissemination might not be apparent. The laparoscopic view enables evaluation of the whole peritoneal cavity to determine whether peritoneal dissemination is present; thus, even if many disseminated nodules are present, the patient could undergo minimallyinvasive surgery and receive systemic chemotherapy, such as sorafenib, as soon as possible. Additionally, Hai et al. (13) reported a case of laparoscopic extirpation of peritoneal dissemination and found that LLR enabled simultaneous extirpation of a solitary nodule for total biopsy.

In conclusion, major LLR may be an option for staged hepatectomy for ruptured HCC controlled after TAE in in properly selected patients.

\section{Acknowledgements}

This study was supported, in part, by a Grant-in-Aid for Scientific Research (KAKENHI) from the Ministry of Health, Labour and Welfare of Japan (16K19935). The funding source had no role in the collection, analysis, or interpretation of the data or in the decision to submit the article for publication.

\section{References}

1 Tanaka S, Kaibori M, Ueno M, Wada H, Hirokawa F, Nakai T, Iida H, Eguchi H, Hayashi M and Kubo S: Surgical outcomes for the ruptured hepatocellular carcinoma: Multicenter analysis with a case-controlled study. J Gastrointest Surg 20(12): 20212034, 2016.

2 Uchiyama H, Minagawa R, Itoh S, Kajiyama K, Harimoto N, Ikegami T, Yoshizumi T, Shirabe K, Takenaka K and Maehara Y: Favorable outcomes of hepatectomy for ruptured hepatocellular carcinoma: Retrospective analysis of primary r0hepatectomized patients. Anticancer Res 36(1): 379-385, 2016.
3 Wakabayashi G, Cherqui D, Geller DA, Buell JF, Kaneko H, Han HS, Asbun H, O'Rourke N, Tanabe M, Koffron AJ, Tsung A, Soubrane O, Machado MA, Gayet B, Troisi RI, Pessaux P, Van Dam RM, Scatton O, Abu Hilal M, Belli G, Kwon CH, Edwin B, Choi GH, Aldrighetti LA, Cai X, Cleary S, Chen KH, Schon MR, Sugioka A, Tang CN, Herman P, Pekolj J, Chen XP, Dagher I, Jarnagin W, Yamamoto M, Strong R, Jagannath P, Lo CM, Clavien PA, Kokudo N, Barkun J and Strasberg SM: Recommendations for laparoscopic liver resection: A report from the second international consensus conference held in morioka. Ann Surg 261(4): 619-629, 2015.

4 Miyamoto M, Sudo T and Kuyama T: Spontaneous rupture of hepatocellular carcinoma: A review of 172 japanese cases. Am J Gastroenterol 86(1): 67-71, 1991.

5 Ikai I, Arii S, Okazaki M, Okita K, Omata M, Kojiro M, Takayasu K, Nakanuma Y, Makuuchi M, Matsuyama Y, Monden M and Kudo M: Report of the 17th nationwide follow-up survey of primary liver cancer in japan. Hepatol Res 37(9): 676-691, 2007.

6 Lee HS, Choi GH, Kang DR, Han KH, Ahn SH, Kim DY, Park JY, Kim SU and Choi JS: Impact of spontaneous hepatocellular carcinoma rupture on recurrence pattern and long-term surgical outcomes after partial hepatectomy. World J Surg 38(8): 20702078, 2014.

7 Aoki T, Kokudo N, Matsuyama Y, Izumi N, Ichida T, Kudo M, $\mathrm{Ku}$ Y, Sakamoto M, Nakashima O, Matsui O and Makuuchi M: Prognostic impact of spontaneous tumor rupture in patients with hepatocellular carcinoma: An analysis of 1160 cases from a nationwide survey. Ann Surg 259(3): 532-542, 2014.

8 Ohtomo K, Furui S, Kokubo T, Yamauchi T, Yashiro N, Itai Y and Iio M: Transcatheter arterial embolization for spontaneous rupture of hepatocellular carcinoma. Radiat Med 6(4): 150-156, 1988.

9 Nakahira S, Takeda Y, Katsura Y, Kato T, Hatanaka N and Tamura S: Laparoscopic left hepatectomy with tumor thrombectomy in patients with hepatocellular carcinoma concomitant with advanced portal vein tumor thrombus. Surg Endosc 28(12): 3505, 2014.

10 Zhang CW, Liu J, Hong DF, Wang ZF, Hu ZM, Huang DS, Shang MJ and Yao WF: Pure laparoscopic radical resection for type iiia hilar cholangiocarcinoma. Surg Endosc, 2017. doi: 10.1007/s00464-017-5741-4. [Epub ahead of print]

11 Xu HW, Liu F, Li HY, Wei YG and Li B: Outcomes following laparoscopic versus open major hepatectomy for hepatocellular carcinoma in patients with cirrhosis: A propensity score-matched analysis. Surg Endosc 32(2): 712-719, 2017.

12 Noda T, Eguchi H, Wada H, Iwagami Y, Yamada D, Asaoka T, Gotoh K, Kawamoto K, Takeda Y, Tanemura M, Umeshita K, Doki Y and Mori M: Short-term surgical outcomes of minimally invasive repeat hepatectomy for recurrent liver cancer. Surg Endosc 32(1): 46-52, 2017.

13 Hai S, Okada T, Iimuro Y, Hirano T, Suzumura K and Fujimoto J: Successful laparoscopic extirpation of peritoneal dissemination after hepatectomy for ruptured hepatocellular carcinoma. Asian J Endosc Surg 8(4): 465-467, 2015.

Received December 24, 2017

Revised January 30, 2018

Accepted February 6, 2018 